\title{
Facile synthesis of optically active oxindols by copper-catalyzed asymmetric monotosylation of prochiral 1,3-diols
}

\author{
Masami Kuriyama, Satoko Tanigawa, Yuki Kubo, Yosuke Demizu, and Osamu Onomura* \\ Graduate School of Biomedical Sciences, Nagasaki University, \\ 1-14 Bunkyo-machi, Nagasaki 852-8521, Japan \\ onomura@nagasaki-u.ac.jp
}

\begin{abstract}
The facile synthetic method of optically active 3,3-disubstituted oxindoles with excellent enantioselectivity was achieved using chiral copper-catalyzed desymmetrization of prochiral 1,3-diols. The monotosylated product was transformed into oxindole derivatives efficiently.
\end{abstract}

Dedicated to Professor Dr. Henri Kagan on his $80^{\text {th }}$ birthday

\section{Introduction}

Oxindols are important heterocycles found in various naturally occurring compounds and biologically active molecules. ${ }^{1}$ Particularly, numerous methods have been reported for the enantioselective synthesis of oxindoles including Heck reaction ${ }^{2}$, cyanoamidation ${ }^{3}$, cycloaddition $^{4}$, alkylation ${ }^{5}$, arylation ${ }^{6}, 1,2-$ addition $^{7}, 1,4$-addition ${ }^{8}$, hydroxylation ${ }^{9}$, fluorination ${ }^{10}$, rearrangement ${ }^{11}$, and addition to isatins ${ }^{12}$. In addition, enzyme-catalyzed asymmetric desymmetrization was known as an effective synthetic method for optically active oxindoles bearing a quaternary stereocenter at the 3-position, ${ }^{13}$ but nonenzymic methods have not been reported. Recently, we have developed chiral copper-catalyzed kinetic resolution and desymmetrization of diols (Scheme 1). ${ }^{14,15}$ Herein, we would like to describe the efficient synthesis of optically active 3,3-disubstituted oxindoles using chiral copper-catalyzed asymmetric monotosylation $^{15 \mathrm{e}}$ of prochiral 3,3-bis(hydroxymethyl)oxindoles. 
Scheme 1. Asymmetric benzoylation of 1,2-diols with $\mathrm{Cu}(\mathrm{II})-(R, R)-\mathrm{Ph}-\mathrm{BOX}$

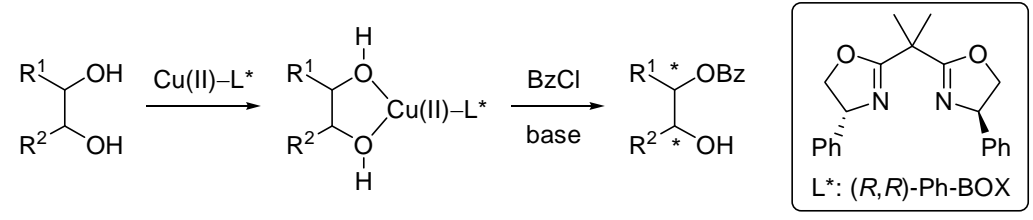

\section{Results and Discussion}

First of all, the influence of $N$-protecting groups in 3,3-bis(hydroxymethyl)oxindoles 1 was examined in chiral copper-catalyzed desymmetrization with tosyl chloride (Table 1). The reaction using $N$-Boc oxindole 1a did not proceed (entry 1 ). $N$-Acetyloxindole $\mathbf{1 b}$ gave the desired product $\mathbf{2 b}$ with $9 \%$ yield and $63 \%$ ee (entry 2). $N$-Methoxymethyloxindole 1c improved enatioselectivity up to $99 \%$ ee though chemical yield was still low (entry 3 ). In the case of $N$-methyloxindole $\mathbf{1 d}$, the product $\mathbf{2 d}$ was obtained in $75 \%$ yield and $98 \%$ ee (entry 4 ). Although $N$-benzyloxindole 1e led to moderate yield keeping $99 \%$ ee, $N$-( $p$-methylbenzyl)oxindole $\mathbf{1 f}$ decreased both chemical yield and enantioselectivity (entries 5-6).

Table 1. Copper-catalyzed asymmetric monotosylation of 3,3-bis(hydroxymethyl)oxindoles ${ }^{a}$

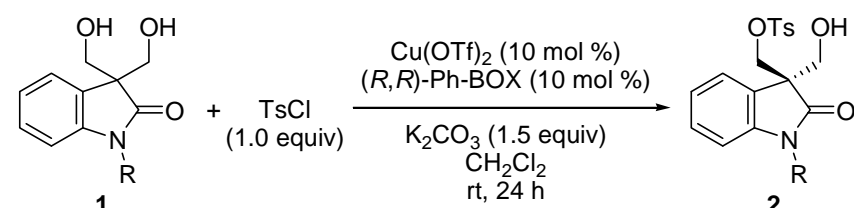

\begin{tabular}{cccccc}
\hline entry & substrate & R & product & $\begin{array}{c}\text { yield } \\
(\%)^{b}\end{array}$ & $\begin{array}{c}\text { ee } \\
(\%)^{c}\end{array}$ \\
\hline 1 & 1a & Boc & 2a & 0 & - \\
2 & 1b & Ac & 2b & 9 & 63 \\
3 & 1c & MOM & 2c & 20 & 99 \\
4 & 1d & Me & 2d & 75 & 98 \\
5 & 1e & Bn & 2e & 54 & 99 \\
6 & 1f & $p-\mathrm{MeBn}$ & $\mathbf{2 f}$ & 19 & 37
\end{tabular}

\footnotetext{
${ }^{a}$ Reaction conditions: 3,3-bis(hydroxymethyl)oxindole $1(0.5 \mathrm{mmol})$, p-toluenesulfonyl chloride $(0.5 \mathrm{mmol}), \mathrm{K}_{2} \mathrm{CO}_{3}(0.75$
} $\mathrm{mmol}), \mathrm{Cu}(\mathrm{OTf})_{2}(10 \mathrm{~mol} \%),(R, R)-\mathrm{Ph}-\mathrm{BOX}(10 \mathrm{~mol} \%), \mathrm{CH}_{2} \mathrm{Cl}_{2}(2 \mathrm{~mL}), \mathrm{rt}, 24 \mathrm{~h} .{ }^{b}$ Isolated yield. ${ }^{c}$ Determined by HPLC 
Then, we tried the transformation of monotosylated oxindole 2d (Scheme 2). In the synthesis of 2d using 1.2 equiv of tosyl chloride, chemical yield and enantioselectivity were improved to give the product in $81 \%$ yield and $99 \%$ ee. Monotosylated oxindole 2d was benzylated with benzyl 2,2,2trichloroacetimidate under acidic condition. The crude product of $\mathbf{3}$ was used for cyanation without further purification to give the compound 4 with $76 \%$ yield ( 2 steps). The chiral oxindole derivatives bearing 3-cyanomethyl group are known as useful synthetic intermediates. ${ }^{3}$

Scheme 2. Transformation of (R)-(+)-3-hydroxymethyl-1-methyl-3-(p-toluenesulfonyloxymethyl)oxindole 2d
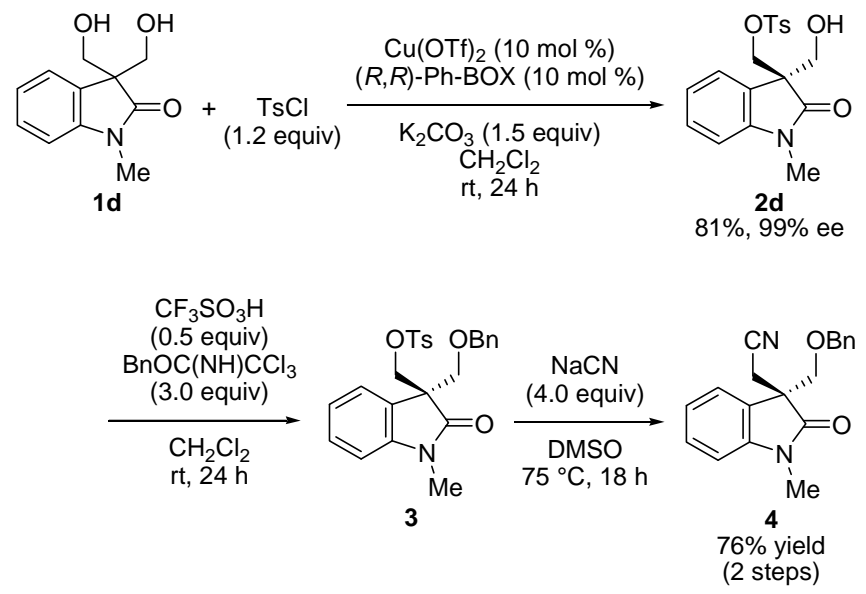

The absolute configuration of $\mathbf{2 d}$ was determined by X-ray crystallographic analysis. ${ }^{16}$ Monotosylated oxindole 2d was treated with (+)-10-camphorsulfonyl chloride, giving the sulfonate ester $\mathbf{5}$, which was recrystallized from diethyl ether to provide fine crystals after purification with silica gel column chromatography (Scheme 3). Then, the X-ray crystallographic analysis of the sulfonate ester 5 proved that the newly generated stereogenic center was $R$ (Figure 1). 
Scheme 3. Synthesis of $(R)-(+)-3-((+)-10$-camphorsulfonyloxymethyl)-1-methyl-3-(p-toluenesulfonyloxymethyl)oxindole 5

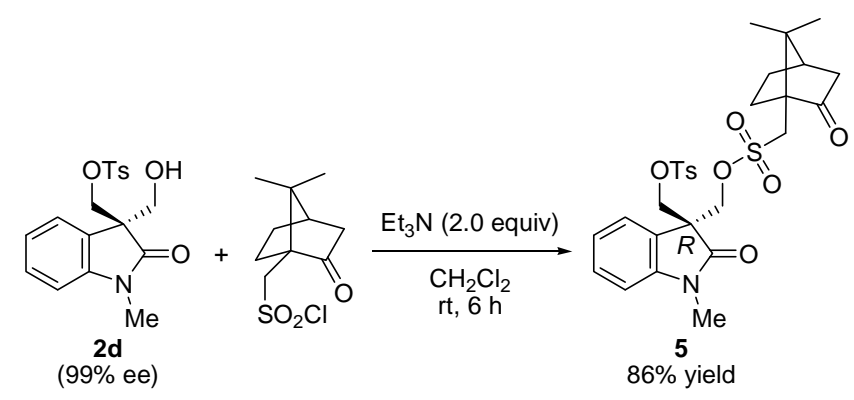

Figure 1. X-ray crystal structure of $(R)-(+)-3-((+)-10$-camphorsulfonyloxymethyl)-1-methyl-3- $(p-$ toluenesulfonyloxymethyl)oxindole 5 .

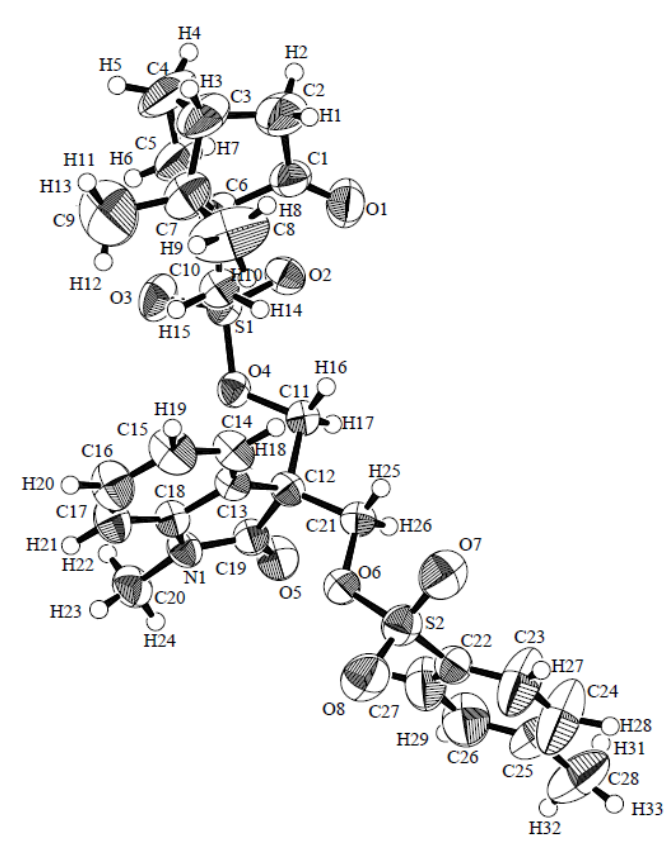

\section{Conclusion}

In summary, the enantioselective synthesis of oxindoles bearing a quaternary stereocenter at the 3position was developed using copper-catalyzed asymmetric monotosylation of prochiral 1,3-diols. The reaction with $\mathrm{N}$-methyl oxindole 1d led to excellent yield and enantioselectivity. The tosylated product was readily converted to the oxindole derivatives bearing 3-cyanomethyl group via benzylaition and 
cyanation. The absolute configuration of monotosylated oxindole 2d was determined by the X-ray crystallographic analysis of the sulfonate ester 5.

\section{Experimental}

\subsection{General}

All melting points are not corrected. IR spectra were obtained with Shimadzu FT-IR-8100 and expressed in $\mathrm{cm}^{-1} .{ }^{1} \mathrm{H}$ and ${ }^{13} \mathrm{C}$ NMR spectra were taken with Varian Gemini 300 or JEOL JNM-AL 400, and chemical shift values are expressed in ppm relative to internal TMS. Abbreviations are as follows: s, singlet; d, doublet; t, triplet; q, quartet; m, multiplet; br, broad. High-resolution mass spectra (HRMS) were recorded with JEOL JMS-700N spectrometer. Specific rotations were measured with JASCO DIP1000. All reagents and solvents were used as received without further purification. The products were isolated by silica gel column chromatography with Merck silica gel 60 .

\subsection{General procedure of Copper-catalyzed asymmetric monotosylation of 3,3-bis(hydroxyl-}

\section{methyl)oxindoles}

To a solution of $\mathrm{Cu}(\mathrm{OTf})_{2}(0.05 \mathrm{mmol}, 18.1 \mathrm{mg})$ and $(R, R)-\mathrm{Ph}-\mathrm{BOX}(0.05 \mathrm{mmol}, 16.7 \mathrm{mg})$ in $\mathrm{CH}_{2} \mathrm{Cl}_{2}$ $(2 \mathrm{~mL})$ were added 3,3-bis(hydroxymethyl)oxindoles $1 \mathbf{e}(0.5 \mathrm{mmol}, 89.6 \mathrm{mg}), \mathrm{K}_{2} \mathrm{CO}_{3}(0.75 \mathrm{mmol}, 104$ $\mathrm{mg}$ ), and $p$-toluenesulfonyl chloride $(0.6 \mathrm{mmol}, 114 \mathrm{mg})$. After stirring for $24 \mathrm{~h}$ at room temperature, water $(10 \mathrm{~mL})$ was added. The resulting mixture was extracted with AcOEt. The combined organic layers were washed with brine, and then dried over $\mathrm{MgSO}_{4}$. Concentration and purification through silica gel column chromatography gave the product.

4.2.1 (+)-1-Acetyl-3-hydroxymethyl-3-(p-toluenesulfonyloxymethyl)oxindole (2b). Yellow oil. IR (neat): $3400,3020,1760,1720,1640,1470 \mathrm{~cm}^{-1} .{ }^{1} \mathrm{H}-\mathrm{NMR}\left(300 \mathrm{MHz}, \mathrm{CDCl}_{3}\right): \delta 8.30-8.15(\mathrm{~m}, 1 \mathrm{H})$, $7.62(\mathrm{~d}, J=7.5 \mathrm{~Hz}, 1 \mathrm{H}), 7.42-7.10(\mathrm{~m}, 6 \mathrm{H}), 4.41(\mathrm{bs}, 2 \mathrm{H}), 3.88-3.81(\mathrm{~m}, 2 \mathrm{H}), 2.72-2.56(\mathrm{~m}, 3 \mathrm{H}), 2.59$ (s, 3H), $2.04(\mathrm{bs}, 1 \mathrm{H}) .{ }^{13} \mathrm{C}-\mathrm{NMR}\left(100 \mathrm{MHz}, \mathrm{CDCl}_{3}\right): \delta 176.5,170.4,145.2,132.0,129.9,129.6,128.9$, 127.9, 125.9, 125.5, 123.4, 116.8, 69.6, 64.6, 54.5, 26.6, 21.7. 63\% ee (HPLC: Daicel chiralcel OG (4.6 $\operatorname{mm} \varphi, 250 \mathrm{~mm}$ ), $n$-Hexane : Isopropanol = $10: 1,254 \mathrm{~nm}, 1.0 \mathrm{ml} / \mathrm{min}, 64 \mathrm{~min}$ and $77 \mathrm{~min}$ (enriched)) 
$[\alpha]^{19}{ }_{\mathrm{D}}+47.0\left(\right.$ c 1.0, $\left.\mathrm{CHCl}_{3}\right)$. MS [HR-FAB $\left.(+)\right]: \mathrm{m} / \mathrm{z}$ calcd for $\mathrm{C}_{19} \mathrm{H}_{20} \mathrm{O}_{6} \mathrm{NS}[\mathrm{M}+\mathrm{H}]^{+} 390.1011$, found 390.1044 .

\subsection{2 (+)-3-Hydroxymethyl-1-methoxymethyl-3-(p-toluenesulfonyloxymethyl)oxindole (2c).}

Colorless oil. IR (neat): 3400, 2940, 1730, $1620 \mathrm{~cm}^{-1} .{ }^{1} \mathrm{H}-\mathrm{NMR}\left(400 \mathrm{MHz}, \mathrm{CDCl}_{3}\right): \delta 7.61(\mathrm{~d}, J=8.3$ $\mathrm{Hz}, 2 \mathrm{H}), 7.38-7.29(\mathrm{~m}, 4 \mathrm{H}), 7.10-7.02(\mathrm{~m}, 2 \mathrm{H}), 5.15-5.05(\mathrm{~m}, 2 \mathrm{H}), 4.46(\mathrm{~d}, J=9.3 \mathrm{~Hz}, 1 \mathrm{H}), 4.37(\mathrm{~d}, J=$ $9.3 \mathrm{~Hz}, 1 \mathrm{H}), 3.87(\mathrm{~d}, J=11.7 \mathrm{~Hz}, 1 \mathrm{H}), 3.79(\mathrm{~d}, J=11.7 \mathrm{~Hz}, 1 \mathrm{H}), 3.25(\mathrm{~s}, 3 \mathrm{H}), 2.44(\mathrm{~s}, 4 \mathrm{H}) .{ }^{13} \mathrm{C}-\mathrm{NMR}$ $\left(100 \mathrm{MHz}, \mathrm{CDCl}_{3}\right): \delta 176.6,145.0,142.2,132.1,129.8,129.4,127.9,126.0,124.0,123.5,110.1,71.2$ 69.6, 64.1, 56.1, 54.0, 21.6. 99\% ee (HPLC: Daicel chiralcel OJ-H (4.6 mm Isopropanol $=5: 1,254 \mathrm{~nm}, 1.0 \mathrm{ml} / \mathrm{min}, 39 \mathrm{~min}$ and $43 \mathrm{~min}$ (enriched)). $[\alpha]^{20}{ }_{\mathrm{D}}+6.8\left(c 1.0, \mathrm{CHCl}_{3}\right)$. MS [HR-FAB(+)]: m/z calcd for $\mathrm{C}_{19} \mathrm{H}_{22} \mathrm{O}_{6} \mathrm{NS}[\mathrm{M}+\mathrm{H}]^{+}$392.1168, found 392.1193.

4.2.3 (R)-(+)-3-Hydroxymethyl-1-methyl-3-(p-toluenesulfonyloxymethyl)oxindole (2d). White solid of mp 119-120 ${ }^{\circ} \mathrm{C}$. IR (neat): 3350, 3060, 1720, $1620 \mathrm{~cm}^{-1} .{ }^{1} \mathrm{H}-\mathrm{NMR}\left(400 \mathrm{MHz}, \mathrm{CDCl}_{3}\right): \delta 7.67$ (d, $J=8.3 \mathrm{~Hz}, 2 \mathrm{H}), 7.38-7.23(\mathrm{~m}, 4 \mathrm{H}), 7.07(\mathrm{t}, J=7.8 \mathrm{~Hz}, 1 \mathrm{H}), 6.87(\mathrm{~d}, J=7.8 \mathrm{~Hz}, 1 \mathrm{H}), 4.48(\mathrm{~d}, J=9.8$ $\mathrm{Hz}, 1 \mathrm{H}), 4.28(\mathrm{~d}, J=9.8 \mathrm{~Hz}, 1 \mathrm{H}), 3.85(\mathrm{~d}, J=9.2 \mathrm{~Hz}, 1 \mathrm{H}), 3.72(\mathrm{~d}, J=9.2 \mathrm{~Hz}, 1 \mathrm{H}), 3.19(\mathrm{~s}, 3 \mathrm{H}), 2.54$ (bs, 1H), $2.44(\mathrm{~s}, 3 \mathrm{H}) .{ }^{13} \mathrm{C}-\mathrm{NMR}\left(100 \mathrm{MHz}, \mathrm{CDCl}_{3}\right): \delta 175.5,145.0,143.8,132.2,129.8,129.2,127.9$, 126.5 , 124.1, 123.1, 108.6, 69.6, 63.8, 53.2, 26.3, 21.6. 99\% ee (HPLC: Daicel chiralcel OG (4.6 mmp, $250 \mathrm{~mm}$ ), $n$-Hexane : Isopropanol $=10: 1,254 \mathrm{~nm}, 1.0 \mathrm{ml} / \mathrm{min}, 92 \mathrm{~min}$ (enriched) and $98 \mathrm{~min}) .[\alpha]^{22}{ }^{+}$ 17.2 (c 1.0, $\mathrm{CHCl}_{3}$ ). MS [HR-FAB(+)]: m/z calcd for $\mathrm{C}_{18} \mathrm{H}_{20} \mathrm{O}_{5} \mathrm{NS}[\mathrm{M}+\mathrm{H}]^{+} 362.1062$, found 362.1062 .

4.2.4 (+)-1-Benzyl-3-hydroxymethyl-3-(p-toluenesulfonyloxymethyl)oxindole (2e). White solid of mp 123-124 ${ }^{\circ} \mathrm{C}$. IR (neat): 3400, 3010, 17610, $1610 \mathrm{~cm}^{-1} .{ }^{1} \mathrm{H}-\mathrm{NMR}\left(300 \mathrm{MHz}, \mathrm{CDCl}_{3}\right): \delta 7.62(\mathrm{~d}, J=$ 8.4 Hz, 1H), 7.34-7.15 (m, 10H), $7.02(\mathrm{t}, J=7.5 \mathrm{~Hz}, 1 \mathrm{H}), 6.70(\mathrm{~d}, J=7.5 \mathrm{~Hz}, 1 \mathrm{H}), 4.99(\mathrm{~d}, J=15.9 \mathrm{~Hz}$, $1 \mathrm{H}), 4.83(\mathrm{~d}, J=15.9 \mathrm{~Hz}, 1 \mathrm{H}), 4.52(\mathrm{~d}, J=9.3 \mathrm{~Hz}, 1 \mathrm{H}), 4.41(\mathrm{~d}, J=9.3 \mathrm{~Hz}, 1 \mathrm{H}), 3.92(\mathrm{~d}, J=11.1 \mathrm{~Hz}$, $1 \mathrm{H}), 3.80(\mathrm{~d}, J=11.1 \mathrm{~Hz}, 1 \mathrm{H}), 2.47$ (brs, 1H), $2.43(\mathrm{~s}, 3 \mathrm{H}) .{ }^{13} \mathrm{C}-\mathrm{NMR}\left(100 \mathrm{MHz}, \mathrm{CDCl}_{3}\right): \delta 175.7$, $144.9,143.0,135.0,132.1,129.8,129.1,128.8,127.9,127.6,126.9,126.5,123.9,123.1,109.6,69.8$, 64.0, 53.5, 43.7, 21.6. 99\% ee (HPLC: Daicel chiralcel OG (4.6 mm $\varphi, 250 \mathrm{~mm})$, n-Hexane : 
Isopropanol $=10: 1,254 \mathrm{~nm}, 1.0 \mathrm{ml} / \mathrm{min}, 60 \mathrm{~min}$ and $65 \mathrm{~min}($ enriched $)) \cdot[\alpha]^{22}+29.5\left(c 1.0, \mathrm{CHCl}_{3}\right)$. MS [HR-FAB(+)]: m/z calcd for $\mathrm{C}_{24} \mathrm{H}_{24} \mathrm{O}_{5} \mathrm{NS}[\mathrm{M}+\mathrm{H}]^{+}$438.1375, found 438.1375.

\subsection{5 (-)-3-Hydroxymethyl-1-(p-methylbenzyl)-3-(p-toluenesulfonyloxymethyl)oxindole}

(2f). Yellow oil. IR (neat): $\quad 3360,2920,1700,1620,1470 \mathrm{~cm}^{-1} .{ }^{1} \mathrm{H}-\mathrm{NMR}\left(300 \mathrm{MHz}, \mathrm{CDCl}_{3}\right): \delta 7.60(\mathrm{~d}, J=$ 8.1 Hz, 1H), 7.18-7.06 (m, 9H), $6.98(\mathrm{t}, J=7.8 \mathrm{~Hz}, 1 \mathrm{H}), 6.69(\mathrm{~d}, J=7.8 \mathrm{~Hz}, 1 \mathrm{H}), 4.93(\mathrm{~d}, J=15.6 \mathrm{~Hz}$ 1H), $4.74(\mathrm{~d}, J=15.6 \mathrm{~Hz}, 1 \mathrm{H}), 4.52(\mathrm{~d}, J=9.6 \mathrm{~Hz}, 1 \mathrm{H}), 4.40(\mathrm{~d}, J=9.6 \mathrm{~Hz}, 1 \mathrm{H}), 3.90-3.72(\mathrm{~m}, 2 \mathrm{H})$, $2.42(\mathrm{~s}, 3 \mathrm{H}), 2.29(\mathrm{~s}, 3 \mathrm{H}), 1.50(\mathrm{bs}, 1 \mathrm{H}) .{ }^{13} \mathrm{C}-\mathrm{NMR}\left(100 \mathrm{MHz}, \mathrm{CDCl}_{3}\right): \delta 175.7,144.9,143.0,137.3$, $132.1,131.9,129.8,129.5,129.1,127.9,126.8,123.9,123.0,109.7,69.8,63.9,53.5,43.5,21.6,21.0$. 37\% ee (HPLC: Daicel chiralcel AS (4.6 mmp, $250 \mathrm{~mm}), n$-Hexane : Isopropanol $=10: 1,254 \mathrm{~nm}, 1.0$ $\mathrm{ml} / \mathrm{min}, 17 \mathrm{~min}$ and $26 \mathrm{~min}$ (enriched)). $[\alpha]^{19}{ }_{\mathrm{D}}-0.8\left(\right.$ c $\left.0.9, \mathrm{CHCl}_{3}\right)$. MS $[\mathrm{HR}-\mathrm{FAB}(+)]: \mathrm{m} / \mathrm{z}$ calcd for $\mathrm{C}_{25} \mathrm{H}_{26} \mathrm{O}_{5} \mathrm{NS}[\mathrm{M}+\mathrm{H}]^{+}$452.1532, found 452.1525.

\subsection{Transformation of $(R)-(+)-3-h y d r o x y m e t h y l-1-m e t h y l-3-(p$-toluenesulfonyloxymethyl)-}

\section{oxindole (2d)}

4.3.1. (R)-(+)-3-Benzyloxymethyl-3-cyanomethyl-1-methyloxindole (4). To a solution of $2 \mathrm{~d}$ (0.8 $\mathrm{mmol})$ in $\mathrm{CH}_{2} \mathrm{Cl}_{2}(3 \mathrm{~mL})$ was added $\mathrm{BnOC}(\mathrm{NH}) \mathrm{CCl}_{3}(2.4 \mathrm{mmol})$ at $0{ }^{\circ} \mathrm{C}$. Then, $\mathrm{CF}_{3} \mathrm{SO}_{3} \mathrm{H}(0.4 \mathrm{mmol})$ in $\mathrm{CH}_{2} \mathrm{Cl}_{2}(1 \mathrm{~mL})$ was added slowly at $0{ }^{\circ} \mathrm{C}$, and the reaction mixture was stirred at room temperature for 24 h. Saturated $\mathrm{NH}_{4} \mathrm{Cl}$ was added and the resulting mixture was extracted with AcOEt. The combined organic layers were dried over $\mathrm{MgSO}_{4}$ and filtration and concentration gave the crude product of $(R)-3$ benzyloxymethyl-1-methyl-3-(p-toluenesulfonyloxymethyl)oxindole 3, which was used without further purification.

After stirred at room temperature for $10 \mathrm{~min}$, the mixture of $\mathrm{NaCN}(3.2 \mathrm{mmol})$ and crude product 3 was stirred at $75^{\circ} \mathrm{C}$ for $18 \mathrm{~h}$. Then, cold water and ether were added at room temperature. The resulting mixture was extracted with ether and the aqueous layer was treated with $20 \% \mathrm{FeSO}_{4}$. The combined organic layers were washed with cold water and brine, and dried over $\mathrm{MgSO}_{4}$. Concentration and purification through silica gel column chromatography gave (R)-(+)-3-benzyloxymethyl-3- 
cyanomethyl-1-methyloxindole 4 in 76\% yield (2 steps). Yellow oil. IR (neat): $2250,1620,1260 \mathrm{~cm}^{-1}$. ${ }^{1} \mathrm{H}-\mathrm{NMR}\left(300 \mathrm{MHz}, \mathrm{CDCl}_{3}\right): \delta$ 7.38-6.92 (m, 9H), $4.50(\mathrm{~d}, J=2.7 \mathrm{~Hz}, 2 \mathrm{H}), 3.80(\mathrm{~d}, J=9.0 \mathrm{~Hz}, 1 \mathrm{H})$, $3.62(\mathrm{~d}, J=9.0 \mathrm{~Hz}, 1 \mathrm{H}), 3.23(\mathrm{~s}, 3 \mathrm{H}), 3.04(\mathrm{~d}, J=16.5 \mathrm{~Hz}, 1 \mathrm{H}), 2.76(\mathrm{~d}, J=16.5 \mathrm{~Hz}, 1 \mathrm{H}) .{ }^{13} \mathrm{C}-\mathrm{NMR}$ $\left(100 \mathrm{MHz}, \mathrm{CDCl}_{3}\right): \delta 175.0,143.5,137.3,129.4,128.4,128.2,127.8,127.5,124.3,123.1,116.2,108.6$, 73.7, 72.2, 49.8, 26.5, 22.2. $[\alpha]^{22}{ }_{\mathrm{D}}+16.7\left(c \mathrm{c} 0.36, \mathrm{CH}_{2} \mathrm{Cl}_{2}\right)$. MS [HR-EI(+)]: m/z calcd for $\mathrm{C}_{19} \mathrm{H}_{18} \mathrm{~N}_{2} \mathrm{O}_{2}$ $[\mathrm{M}]^{+}$306.1368, found 306.1368.

\subsection{2. (R)-(+)-3-((+)-10-Camphorsulfonyloxymethyl)-1-methyl-3-(p-toluenesulfonyloxymethyl)-}

oxindole (5). To a solution of $\mathbf{2} \mathbf{d}(0.5 \mathrm{mmol})$ in $\mathrm{CH}_{2} \mathrm{Cl}_{2}(3 \mathrm{~mL})$ was added triethylamine $(1.0 \mathrm{mmol})$ and (+)-10-camphorsulfonyl chloride $(0.6 \mathrm{mmol})$ and the mixture was stirred at room temperature for $6 \mathrm{~h}$. Then, saturated $\mathrm{NaHCO}_{3}$ was added, and the resulting mixture was extracted with ethyl acetate. The combined organic layers were dried over $\mathrm{MgSO}_{4}$ and concentration and purification through silica gel column chromatography gave $(R)-(+)-3-((+)-10$-camphorsulfonyloxymethyl)-1-methyl-3-(p-toluenesulfonyloxymethyl)oxindole 5 in $86 \%$ yield. This product was recrystallized from diethyl ether, and Xray crystallographic analysis was conducted. Colorless plates of mp 109-110 ${ }^{\circ} \mathrm{C}$. IR (neat): 2960,1750 , 1710, 1610, 1360, $1180 \mathrm{~cm}^{-1} .{ }^{1} \mathrm{H}-\mathrm{NMR}\left(300 \mathrm{MHz}, \mathrm{CDCl}_{3}\right): \delta 7.68(\mathrm{~d}, J=8.4 \mathrm{~Hz}, 2 \mathrm{H}), 7.37-7.31(\mathrm{~m}$, 4H), 7.09-7.04 (m, 1H), 6.87-6.84 (m, 1H), 4.57 (d, $J=9.9 \mathrm{~Hz}, 1 \mathrm{H}), 4.41(\mathrm{~d}, J=9.9 \mathrm{~Hz}, 1 \mathrm{H}), 4.34(\mathrm{~d}, J$ $=9.6 \mathrm{~Hz}, 1 \mathrm{H}), 4.18(\mathrm{~d}, J=9.6 \mathrm{~Hz}, 1 \mathrm{H}), 3.39(\mathrm{~d}, J=15.0 \mathrm{~Hz}, 1 \mathrm{H}), 3.21(\mathrm{~s}, 3 \mathrm{H}), 2.84(\mathrm{~d}, J=15.0 \mathrm{~Hz}$, $1 \mathrm{H}), 2.45(\mathrm{~s}, 3 \mathrm{H}), 2.37-2.17(\mathrm{~m}, 2 \mathrm{H}), 2.07(\mathrm{t}, J=4.5 \mathrm{~Hz}, 1 \mathrm{H}), 2.01-1.93(\mathrm{~m}, 1 \mathrm{H}), 1.87(\mathrm{~d}, J=18.6 \mathrm{~Hz}$ 1H), 1.47-1.31 (m, 2H), $1.01(\mathrm{~s}, 3 \mathrm{H}), 0.80(\mathrm{~s}, 3 \mathrm{H}) .{ }^{13} \mathrm{C}-\mathrm{NMR}\left(100 \mathrm{MHz}, \mathrm{CDCl}_{3}\right): \delta 213.7,172.5,145.2$, $143.8,131.9,129.9,129.6,127.9,125.3,124.8,123.1,108.6,77.2,69.4,68.8,57.6,51.7,47.9,47.2$, $42.6,42.3,26.8,26.5,24.7,21.6,19.54,19.49 .[\alpha]^{22}+24.0\left(c 0.5, \mathrm{CHCl}_{3}\right) . \mathrm{MS}[\mathrm{HR}-\mathrm{FAB}(+)]: \mathrm{m} / \mathrm{z}$ calcd for $\mathrm{C}_{28} \mathrm{H}_{34} \mathrm{O}_{8} \mathrm{NS}_{2}[\mathrm{M}+\mathrm{H}]^{+}$576.1720, found 576.1752.

\section{Acknowledgments}

This work was supported by the president's discretion fund of Nagasaki University. 
(1) (a) Marti, C.; Carreira, E. M. Eur. J. Org. Chem. 2003, 2209-2219. (b) Galliford, C. V.; Scheidt, K. A. Angew. Chem. Int. Ed. 2007, 46, 8748-8758.

(2) (a) Ashimori, A.; Buchand, B.; Overman, L. E.; Poon, D. J. J. Am. Chem. Soc. 1998, 120, 64776487. (b) Dounay, A. B.; Hatanaka, K.; Kodanko, J. J.; Oestreich, M.; Overman, L. E.; Pfeifer, L. A.; Weiss, M. M. J. Am. Chem. Soc. 2003, 125, 6261-6271. (c) Busacca, C. A.; Grossbach, D.; So, R. C.; O’Brien, E. M.; Spinelli, E. M. Org. Lett. 2003, 5, 595-598. (d) McDermott, M. C.; Stephenson, R.; Walkington, A. Synlett 2007, 51-54. (e) Pinto, A.; Jia, Y.; Neuville, L.; Zhu, J. Chem. Eur. J. 2007, 13, 961-967.

(3) (a) Yasui, Y.; Kamisaki, H.; Takemoto, Y. Org. Lett. 2008, 10, 3303-3306. (b) Reddy V. J.; Douglas C. J. Org. Lett. ASAP.

(4) Trost, B. M.; Cramer, N.; Silverman, S. M. J. Am. Chem. Soc. 2007, 129, 12396-12397.

(5) (a) Lee, T. B. K.; Wong, G. S. K. J. Org. Chem. 1991, 56, 872-875. (b) Trost, B. M.; Frederiksen, M. U. Angew. Chem. Int. Ed. 2005, 44, 308-310. (c) Trost, B. M.; Zhang, Y. J. Am. Chem. Soc. 2007, 129, 14548-14549. (d) Ma, S.; Han, X.; Krishnan, S.; Virgil, S. C.; Stoltz, B. M. Angew. Chem. Int. Ed. 2009, 48, 8037-8041.

(6) (a) Lee, S.; Hartwig, J. F. J. Org. Chem. 2001, 66, 3402-3415. (b) Kündig, E. P.; Seidel, T. M.; Jia, Y.-X.; Bernardinelli, G. Angew. Chem. Int. Ed. 2007, 46, 8484-8487. (c) Jia, Y.-X.; Hillgren, J. M.; Watson, E. L.; Marsden, S. P.; Kündig, E. P. Chem. Commun. 2008, 4040-4042. (d) Luan, X.; Mariz, R.; Robert, C.; Gatti, M.; Blumentritt, S.; Linden, A.; Dorta, R. Org. Lett. 2008, 10, 55695572. (e) Taylor, A. M.; Altman, R. A.; Buchwald, S. L. J. Am. Chem. Soc. 2009, 131, 9900-9901.

(7) (a) Ogawa, S.; Shibata, N.; Inagaki, J.; Nakamura, S.; Toru, T.; Shiro, M. Angew. Chem. Int. Ed. 2007, 46, 8666-8669. (b) Tian, X.; Jiang, K.; Peng, J.; Du, W.; Chen, Y.-C. Org. Lett. 2008, 10, 3583-3586. (c) Qian, Z.-Q.; Zhou, F.; Du, T.-P.; Wang, B.-L.; Ding, M.; Zhao, X.-L.; Zhou, J. 
Chem. Commun. 2009, 6753-6755. (d) Cheng, L.; Liu, L.; Jia, H.; Wang, D.; Chen, Y.-J. J. Org. Chem. 2009, 74, 4650-4653. (e) Bui, T.; Borregan, M.; Barbas, C. F. III J. Org. Chem. 2009, 74, 8935-8938. (f) Bravo, N.; Mon, I.; Companyó, X.; Alba, A.-N.; Moyano, A.; Rios, R. Tetrahedron Lett. 2009, 50, 6624-6626. (g) Mouri, S.; Chen, Z.; Mitsunuma, H.; Furutachi, M.; Matsunaga, S.; Shibasaki, M. J. Am. Chem. Soc. 2010, 132, 1255-1257.

(8) (a) Bui, T.; Syed, S.; Barbas, C. F. III J. Am. Chem. Soc. 2009, 131, 8758-8759. (b) Kato, Y.; Furutachi, M.; Chen, Z.; Mitsunuma, H.; Matsunaga, S.; Shibasaki, M. J. Am. Chem. Soc. 2009, 131, 9168-9169. (c) He, R.; Ding, C.; Maruoka, K. Angew. Chem. Int. Ed. 2009, 48, 4559-4561. (d) Galzerano, P.; Bencivenni, G.; Pesciaioli, F.; Mazzanti, A.; Giannichi, B.; Sambri, L.; Bartoli, G.; Melchiorre, P. Chem. Eur. J. 2009, 15, 7846-7849.

(9) (a) Ishimaru, T.; Shibata, N.; Nagai, J.; Nakamura, S.; Toru, T.; Kanemasa, S. J. Am. Chem. Soc. 2006, 128, 16488-16489. (b) Sano, D.; Nagata, K.; Itoh, T. Org. Lett. 2008, 10, 1593-1595.

(10) (a) Hamashima, Y.; Suzuki, T.; Takano, H.; shimura, Y.; Sodeoka, M. J. Am. Chem. Soc. 2005, 127, 10164-10165. (b) Ishimaru, T.; Shibata, N.; Horikawa, T.; Yasuda, N.; Nakamura, S.; Toru, T.; Shiro, M. Angew. Chem. Int. Ed. 2008, 47, 4157-4161.

(11) (a) Shaw, S. A.; Aleman, P.; Vedejs, E. J. Am. Chem. Soc. 2003, 125, 13368-13369. (b) Hills, I.; Fu, G. C. Angew. Chem. Int. Ed. 2003, 42, 3921-3924. (c) Linton, E. C.; Kozlowski, M .C. J. Am. Chem. Soc. 2008, 130, 16162-16163. (d) Duffey, T. A.; Shaw, S. A.; Vedejs, E. J. Am. Chem. Soc. 2009, 131, 14-15.

(12) (a) Luppi, G.; Cozzi, P. G.; Monari, M.; Kaptein, B.; Broxterman, Q. B.; Tomasini, C. J. Org. Chem. 2005, 70, 7418-7421. (b) Shintani, R.; Inoue, M.; Hayashi, T. Angew. Chem. Int. Ed. 2006, 45, 3353-3356. (c) Toullec, P. Y.; Jagt, R. B. C.; de Vries, J. G.; Feringa, B. L.; Minnaard, A. J. Org. Lett. 2006, 8, 2715-2718. (d) Chen, J.-R.; Liu, X.-P.; Zhu, X.-Y.; Li, L.; Qian, T.-F.; Zhang, 
J.-M.; Xiao, W.-J. Tetrahedron, 2007, 63, 10437-10444. (e) Lai, H.; Huang, Z.; Wu, Q.; Qin, Y. J. Org. Chem. 2009, 74, 283-288. (f) Tomita, D.; Yamatsugu, K.; Kanai, M.; Shibasaki, M. J. Am. Chem. Soc. 2009, 131, 6946-6948. (g) Angelici, G.; Corrêa, R. J.; Garden, S. J.; Tomasini, C. Tetrahedron Lett. 2009, 50, 814-817. (h) Qiao, X.-C.; Zhu, S.-F.; Zhou, Q.-L. Tetrahedron: Asymmetry 2009, 20, 1254-1261.

(13) (a) Nakazawa, K.; Hayashi, M.; Tanaka, M.; Aso, M.; Suemune, H. Tetrahedron: Asymmetry 2001, 12, 897-901. (b) Akai, S.; Tsujino, T.; Akiyama, E.; Tanimoto, K.; Naka, T.; Kita, Y. J. Org. Chem. 2004, 69, 2478-2486.

(14) Review: Matsumura, Y.; Onomura, O.; Demizu, Y. Yuki Gosei Kagaku Kyokaishi 2007, 65, 216225.

(15)(a) Matsumura, Y.; Maki, T.; Murakami, S.; Onomura, O. J. Am. Chem. Soc. 2003, 125, 2052-2053. (b) Mitsuda, M.; Tanaka, T.; Tanaka, T.; Demizu, Y.; Onomura, O.; Matsumura, Y. Tetrahedron Lett. 2006, 47, 8073-8077. (c) Matsumoto, K.; Mitsuda, M.; Ushijima, N.; Demizu, Y.; Onomura, O. Matsumura, Y. Tetrahedron Lett. 2006, 47, 8453-8456. (d) Onomura, O.; Arimoto, H.; Matsumura, Y.; Demizu, Y. Tetrahedron Lett. 2007, 48, 8668-8672. (e) Demizu, Y.; Kubo, Y.; Matsumura, Y.; Onomura, O. Synlett 2008, 433-437.

(16) Crystallographic data for $(R)-(+)-3-((+)-10$-camphorsulfonyloxymethyl)-1-methyl-3-(p-toluenesulfonyloxymethyl)oxindole 5 was deposited in the Cambridge Crystallographic Data Centre as supplementary publication number CCDC 767295. Copies of the data can be obtained, free of charge, on application to CCDC, 12 Union Road, Cambridge CB21EZ, UK; fax: +44(0) 1223 336033 or e-mail: deposit@ccdc.cam.ac.uk. 